\title{
Influence of the excessive practice of musical wind instruments as an etiological factor of malocclusion
}

\begin{abstract}
Objective: To evaluate the influence of the excessive practice of wind musical instruments as an etiological factor of malocclusion. The music generated by the wind instruments produces those who listen to different moods, ranging from the mystical to the exhilarated. However, excessive practice of this type of instruments can lead to a number of alterations in the oral cavity in the musician who interprets them. For optimum performance, the instrumentalist must position the instrument in relation to the elements of the stomatognathic apparatus in a specific way, which modifies the natural state of those elements. Performing this action frequently from an early age and for an extended period of time creates a non-physiological oral habit that is an etiological factor of malocclusions, which can have harmful consequences, including the risk of the musician being forced to end his career

Conclusion: After a review of the literature, the main objective was achieved by determining that it is possible that the excessive practice of wind musical instruments is an etiological factor of malocclusion. Also, it becomes imperative the need to go deeper into this topic by designing new research.
\end{abstract}

Keywords: wind instruments, malocclusion, oral habits
Volume 9 Issue 5 - 2018

\author{
William Ubilla Mazzini \\ Dentist, Specialist in Orthodontics, University of Guayaquil, \\ Ecuador
}

Correspondence: William Ubilla Mazzini, Dentist, Specialist in Orthodontics, Pilot School of Dentistry University of Guayaquil, Ecuador, Email williamdt40@hotmail.com

Received: July 13, 2017 | Published: October 17, 2018

\section{Introduction}

Oral habits could be defined as a constant and repetitive practice that is derived from performing an act repetitively using elements of the oral cavity for its execution. This causes abnormal forces to act on the components of the masticatory apparatus, which, for an extended period of time, could cause dentomaxillofacial alterations such as dental malocclusions. ${ }^{1}$ In 1952, Dockrell designed and published a method called "Orthodontic Equation" in which he postulated that various causes acting over a considerable period of time on tissues would produce results. One of these causes is habits, which affect the so-called primary sites: bones of the facial skeleton, teeth, neuromuscular system and soft tissues, except the muscle. Aerophone instruments, also known as wind instruments are those that have the ability to reproduce sounds through the vibrations caused by the air column contained therein. The benefits of playing a musical instrument are several. A 2006 study by McMaster University in Canada indicated that there are changes in the brain anatomy after four months of musical instruction. The best age to start learning a musical instrument is five years. There are several researches that suggest that the practice with wind instruments could cause stomatologic alterations. In 2011, De la Torre and colleagues conducted a study in which they determined that students who played wind instruments were more likely to have malocclusions. There is a great variety of wind instruments, and in each one the musician seeks to accommodate the mouth and its attached structures so that the air circulates in such a way that it can become the expected sound. This allows the performer to perform better.

\section{Etiology of malocclusions}

The development of the face in humans is a highly complex process, but at the same time delicate. This is not only due to growth in size, but involves the maturation of the different anatomical elements that integrate it. The process involves a balance of all the structures that evolve from the child to the adult stage. However, in that long period of time, there are several threats that can alter this development. These alterations can cause various conflicts in the normal function of the elements of the face. The growth and development of the face can be affected due to multiple factors. ${ }^{2}$ One of the consequences of disruption of normal craniofacial development is malocclusion. But to understand the concept of malocclusions we must first define the concept of normocclusion. In 2006, Herrera, Belmonte and Herrera defined normocclusion as "the correct relationship between the upper dental arcade and the lower dental arch, directly related to the harmonious growth of both maxillaries and their osteo mucous structures with the influences of the vegetative neuromuscular system , Respiratory function, swallowing and with the same prevalence of certain vicious habits ". ${ }^{3}$ When there is an alteration in the process of growth and maxillofacial development or the dental location is not what is expected, as a consequence a malocclusion occurs. Malocclusion is one of the biggest facial affections in the world, being the third most common. ${ }^{4}$

1. In a much generalized way, Moyers, in 1996, tells us about five factors that could create malocclusions. These factors are:

2. Inheritance: aberrations of genetic origin are manifested already at birth or several years later, which cause craniofacial malformations that will influence a malpositioned of the teeth.

3. Developmental defects of unknown origin: this term applies to rare cases in which an abnormality in one of the critical stages of development, which is idiopathic, probably occurred.

4. Trauma: There are two types of trauma: prenatal and postnatal. Both can cause dentofacial deformity. It is important to note that the author considers that habits (which are another of the etiological causes of malocclusions) can produce "micro trauma" that occurs over time. 
5. Physical agents: among which are premature loss of primary teeth due to several factors such as caries, etc., and nature of the food in which it indicates that people with a very fibrous diet cause excessive work of the masticatory muscles, Which creates an abnormal force that is exerted on the teeth.

Habits: there are habits that serve as stimuli for normal maxillofacial growth and development, such as natural sucking or chewing. However, there are also abnormal habits that interfere with the normal growth pattern and are involved in the origin of malocclusions. These types of habits are associated with dental malpositions, disturbed breathing habits, suctioning of the thumb and other fingers, difficulties in speech, and even psychological problems such as onychophagia.

\section{Oral habits, characteristics and consequences}

According to Agurto and colleagues, a habit is "the custom or practice acquired by the frequent repetition of the same act, which at first is done consciously and then unconsciously." Some of the habits are classified as non-physiological, for example, finger suction, pacifier, bottle or lip, mouth breathing, lingual interposition at rest and phonoarticulation. ${ }^{6}$

\section{The deformation will depend on three factors:}

1. The age at which the habit begins, being worse the earlier it begins, since the bone structures are in formation, and are more modifiable.

\section{How long the habit lasts.}

3. The frequency, ie, the number of repetitions of the habit per day.

One of the non-physiological habits that may occur is atypical swallowing. In the infantile stage there is a type of swallowing that is not equal to the swallowing that occurs in the adult stage. When infant swallowing persists in an adult, there is talk of atypical swallowing. ${ }^{7}$

\section{Atypical swallowing is characterized by:}

2. When the deglutination phase begins, the tongue is located between the incisors, having contact with the lower lip.

3. During the act of swallowing the tongue is advanced, while the jaw is open.

4. When there is alveolar prognathism in the upper jaw, the lower lip is involved in swallowing, placed between the upper and lower incisors to close the buccal cavity in the anterior part.

5. After this, the swallowing movement occurs with a forced movement of the lower lip.

This has consequences in both the upper and lower jaw, and in the relationship between both. Among them we have:

In the upper jaw:

1. Proclination of the upper incisors.

2. Maxillary prognathism.

3. Alteration of the transverse diameter of the maxilla.

4. Inter incisive diastema.

5. Abnormal position of the tongue located in the anterior part.

In the lower jaw:
1. Retroclination of the lower incisors.

2. Mandibular rotation in its posterior area, increasing the goniaco angle, being able to surpass 140 degrees.

3. Mandibular dysfunction.

In the relationship of both maxillaries:

1. Alterations in the sagittal plane, producing a Class II or Class III malocclusion, depending on the position of the tongue.

2. In the vertical plane, producing an open bite.

3. In the transverse plane, posterior crossbite can occur, depending on the position of the tongue.

4. Other alterations that can be caused are the delay of the eruption of a dental piece, phonetic alterations making difficult the pronunciation of the letters M, P, B, F, V, S. ${ }^{8}$

The mouth breathing is another of the habits that can have negative repercussions provoking disorders of the maxillary morphology, alveodental, and of the craniofacial growth. According to the definition of mouth breathing is "a syndrome that is etiologically diagnosed by obstructive causes, by habits and by anatomy."

In a study carried out in 2013, in children aged 9 to 12 years, the prevalence of certain characteristics was confirmed in those who were mouth breathers, among them: anterior open bite and posterior crossbite, malocclusions, Greater palatine depth, high number of Dola-facial patients. This habit can have consequences as serious as the disturbance of the musculature. Once this occurs, an imbalance occurs in the lips, buccinator muscles and tongue. All these elements begin to act abnormally on the buccal and lingual aspects of the teeth, which gives rise to new and bad positions of the dental pieces, and to a marked affectation in the development of the skull, which is called adenoid fascia or syndrome of the long face. ${ }^{9}$ The most frequent of pathological oral habits is digital suction. This involves inserting a finger into the oral cavity. The etiopathogenesis has not yet been fully defined, however, it is said to be associated with emotional affective disorders and insufficient breastfeeding. This act not only produces the direct pressure of the finger on the arches, but also modify the normal functioning of the perioral and lingual muscles. The anterior open bite in a patient with digital sucking habit is the most common that occurs in those in the stage of growth and development. This habit is characterized by producing anterior open bite, proclination of upper incisors, retroclination of lower incisors, enlargement of the shoulder, class II occlusion, ojival palate and crossbite. ${ }^{10}$

\section{Wind instruments, classification and position required of the elements of the Stomatological apparatus to interpret them}

Wind instruments or aerophones belong to a group of musical instruments that create sound from the vibration produced by the air inside, without involving strings or membranes. The musician is in charge of making the air entering, blowing by means of a nozzle placed in the extremity of the tube of the instrument. These instruments are classified into two main groups: wood and metal. Of these, there are several subclasses according to the type of nozzle that they present. For each one, there is a position of the Stomatological elements that the musician must adopt so that his performance in interpreting it is optimal. They are divided into:

Bevel instruments: this includes flutes and bevel organ tubes. The 
position that must be adopted is to place the mouthpiece on the lower lip while the upper lip is stretched down, forming a small opening. As the tension produced by the upper lip increases or decreases, the different tones are generated.

\section{Single tab mouthpieces}

Simple reed instruments: Clarinets and saxophones are included here. To produce the proper sound, it is necessary that the nozzle is not inserted more than half in the mouth of the instrumentist. While the lower lip surrounds the anterior-inferior teeth, a bed is created on which the bulk of the instrument rests. Light pressure of the anterior superior teeth is required to maintain the nozzle and the upper lip should be sealed.

Double Tongue Instruments: In this group are oboes and bassoons. The upper and lower lips wrap the incisal edges of the upper and lower incisor teeth. A bed is formed in each, in which the nozzle is placed.

\section{Oboe barb mouthpieces}

Instruments of mouth: are the instruments of metal like the trumpet, horn, trombone, tuba, cornet. This group has a nozzle in the shape of a cup, against which the musician supports the lips. The external pterygoid muscle is the one that allows the instrumentalist to protrude the mandible, causing the rim of the cup to be positioned in such a way as to exert pressure against both lips. This force produces a verticalisation in the upper and lower incisors.

Instruments with air reservoir: these instruments can be of two types: with tubes, like the organ, or without tubes, like the accordion.

In general terms, these positions are adopted so that the buccal cavity serves as a tunnel through which air from the lungs is transported to the instrument. The teeth, upper jaw and lower jaw form the frame of reference for the lips, tongue and muscles.

\section{Strayer's classification and its orthodontic implications}

In 1939, Strayer proposed a classification of aerophone instruments according to the type of mouthpiece they had:

Instruments class A: Instruments whose nozzle is cup-shaped, which is placed on both lips when closed. In this case, the lips, incisors, and lower jaw are influenced by forces acting in the lingual direction. The forces generated on these elements are greater than those that occur during swallowing, whistling, or even digital suctioning. To produce the sound, the player must place the jaw in protrusion, so that both lips come into contact with the mouthpiece. This induces anterior mandibular growth. In metal instruments, a negative effect is that it increases the mobility in anterior teeth, being able to lose them when touched with intensity.

Class B instruments: This group has a simple wooden nozzle which is positioned at a certain angle between the upper and lower incisors. The force is applied in the buccal direction in the upper incisors, whereas in the inferior ones the force is towards the lingual. Musicians who use this type of instruments have twice as much retrusion in lower incisors compared to other instruments. The mechanism that is developed with the instruments class B is similar to the one that happens in the habit of digital suction.

Class C instruments: They have a double wooden nozzle that is placed between the upper incisors and the lower incisors. An example is the oboe. The way in which the musician maintains the mouthpiece in the oral cavity causes an increase in the tension in the orbicularis muscle of the lips.

Class D instruments: These have a nozzle in the form of a single aperture. The increased pressure applied by the upper lip on the upper incisors positioned anteriorly, causes their retrusion and reduces the overjet. ${ }^{11}$ Another factor of vital importance to consider is the age at which this practice begins. According to Terán and Castillo, "most professional musicians begin to play an instrument long before their maxillofacial growth and development has ended." The same authors point out that musicians spend several hours in practice to produce music, which is an overload of organs that are not designed to be subjected to these forces continuously. ${ }^{12}$ Also, Alcántara, affirms that the age to which a musician of wind begins to practice oscillates between the 7 or 8 years. ${ }^{13}$ The abuse of wind musical instruments can seriously affect patient occlusion. To orthodontically move a piece requires less than 100 grams of pressure. The force with which the aerophone instruments are supported on the oral tissues can reach the 500 grams of pressure according to measurements made, that is to say, more than five times more than is exerted in a therapeutic way. Effect on the hard and soft oral tissues of different kinds of nozzles and the direction of the forces applied to execute the instruments. A. Clarinete, B. Oboe, C. Trumpet. A study of 91 musicians over two years, whose ages ranged from 11 to 13 years, showed that they maintained differences in the position change of anterior teeth compared to a control group. Salinas in 2002, cites a cephalometric study performed by Parker, with trumpet players who presented class II malocclusion, division 1, that is, with mandibular retroposition. The author noted that the posture adapted by the musicians when playing the instrument, which involved advancing the mandible, could explain the presence of symptoms in the TMJ.14 In a study conducted at the Amadeo Roldán Music Conservatory in 2011, a sample of 52 students was taken. It was obtained that of the 28 students who practiced with a wind instrument, 27 presented malocclusions. Likewise, of the 52 students, 19 presented increased height, 15 presented anterior open bite and 12 , dental crowding. ${ }^{15}$

\section{Conclusion}

A non-physiological oral habit can be defined as a practice that is constantly performed by the repetition of the same act, affecting the elements that make up the oral cavity and altering its normal functioning. However, none of the reviewed bibliographic sources include the practice of wind musical instruments as a non-physiological oral habit. The act of playing an aerophone instrument implies a constant position of the buccal elements, which is not adequate. Also, to achieve perfection the musician requires years of practice, starting at a very young age and employing several hours every day. These factors, as demonstrated in several studies, cause an alteration in maxillofacial structures, generating malocclusions. Therefore, I suggest including the excessive practice of wind instruments as a non-physiological oral habit, being an etiological factor of the malocclusions. The most frequent non-physiological oral habits, such as digital suction, mouth breathing and atypical swallowing, have their own characteristics that generate specific consequences in the stomatognathic system. Some of these characteristics can be replicated when practicing a wind instrument, so there is a possibility of similar consequences that occur in the habits mentioned above. The bibliographic sources in this subject are not abundant, so that, in order to strengthen the proposition that the excessive practice of wind instruments is an etiological factor of the malocclusions, it is necessary to design researches in human 
groups that dedicate themselves to it, applying criteria Inclusion and exclusion. It is also necessary to determine which wind instrument is most injurious when practiced for an extended period of time. Injuries resulting from malocclusions can affect both the oral health and the general health of the patient, which in this case could have a special connotation: they could mean the end of the career of a wind instrumentalist. Therefore, it is of great relevance to determine the way in which these patients can be protected and to prevent damages, without this means abandoning their greatest passion, interpreting music.

\section{Acknowledgement}

None.

\section{Conflict of interest}

The author declares that there is no conflict of interest.

\section{References}

1. Texeira S, Costa Ribero C, Bittercourt M, et al. Defective Oral Habits: Prolonged Sucking. Rev Ciencias da Saude. 1996;152-161.

2. Arocha A, Aranda M, Pérez Y, et al. Malocclusions and deforming oral habits in schoolchildren with early mixed dentition. 2016.

3. Herrera D, Bemonte S, Herrera E. Alterations of maxillofacial development. Prevention of malocclusion. Argentine archives of Pediatrics. 2006.

4. Aguilar M, Villaizá C, Nieto I. Frequency of oral habits etiological factor of malocclusion in the school population. Revista Latinoamericana de Ortodoncia y Odontopediatría. 2009.
5. Moyers R. Manual of Orthodontics. Buenos Aires: Editoria Médica Panamericana. 1996

6. Agurto P, Díaz R, Cádiz O, et al. Frequency of poor oral habits and its association with the development of dentomaxillary abnormalities in children aged 3 to 6 years in the Eastern area of Santiago. Revista Chilena de Pediatría. 1999.

7. Vellini F. Odontologia20. Obtenido de. 2011.

8. Zaldívar L, García S, Expósito I, et al. Abnormal swallowing: some considerations about this habit. Revista Archivo Médico de Camaguey. 2010.

9. Podadera Z, Flores L, Resk A. Impact of mouth breathing on the stomaging system in children aged 9 to 12 years. Revista de Ciencias Médicas. 2013;126-137.

10. Romero M, Romero P, Pardo de Miguel A, et al. Treatment of digital suction in temporary and mixed dentition. Journal of the College of Dentists and Stomatologists of Spain. 2003;77-82.

11. Bluj K, Komarnitki I, Olczak D. Wind instruments and their influence on oral cavity: systematic review. World J Dent. 2014;180-183.

12. Terán H, Castillo A. Effect of the use of wind instruments on dental malocclusions. Review of the literature. Acta Odontológica Venezolana. 2013.

13. Alcántara A. Trumpedland. Obtenido de. 2014.

14. Salinas J. Functional pathology of the stomatognathic system in instrumental musicians. Revista Hospital Clínico Universidad de Chile. 2002;171-178.

15. De la Torre E, Aguirre I, De la Torre J, et al. Stomatological alterations in students of viola, violin and wind instruments. 2013. 